\title{
Some Remarks on the Non-Abelian Fourier Transform in Crossover Designs in Clinical Trials
}

\author{
Peter Zizler \\ Department of Mathematics/Physics and Engineering, Mount Royal University, Calgary, Canada \\ Email: pzizler@mtroyal.ca
}

Received 21 November 2013; revised 21 December 2013; accepted 30 December 2013

Copyright (C) 2014 by author and Scientific Research Publishing Inc.

This work is licensed under the Creative Commons Attribution International License (CC BY).

http://creativecommons.org/licenses/by/4.0/

(c) (i) Open Access

\begin{abstract}
Let $G$ be a non-abelian group and let $l^{2}(G)$ be a finite dimensional Hilbert space of all complex valued functions for which the elements of $G$ form the (standard) orthonormal basis. In our paper we prove results concerning $G$-decorrelated decompositions of functions in $I^{2}(G)$. These $G$ decorrelated decompositions are obtained using the $G$-convolution either by the irreducible characters of the group $G$ or by an orthogonal projection onto the matrix entries of the irreducible representations of the group $G$. Applications of these $G$-decorrelated decompositions are given to crossover designs in clinical trials, in particular the William's $6 \times 3$ design with 3 treatments. In our example, the underlying group is the symmetric group $S_{3}$.
\end{abstract}

\section{Keywords}

Non-Abelian Fourier Transform, Group Algebra, Irreducible Representation, Irreducible Character, $G$-Circulant Matrix, $G$-Decorrelated Decomposition, Crossover Designs in Clinical Trials

\section{Preliminaries}

Consider a finite group $G$, typically non-abelian, and let $\psi$ and $\phi$ be two functions in $l^{2}(G)$, the finite Hilbert space of all complex valued functions (usual inner product $\langle$.$\rangle ) for which elements of G$ form the (standard) basis. We assume that this basis $(G)$ is ordered and make the natural identification, as vector spaces, with $\mathbf{C}^{n}$, where $|G|=n$.

A $G$-convolution of $\psi$ and $\phi$ is defined by the following action, $\sigma \in G$ 


$$
(\psi * \phi)(\sigma)=\sum_{\tau \in G} \psi\left(\sigma \tau^{-1}\right) \phi(\tau) .
$$

Definition. A function $\phi: l^{2}(G) \rightarrow \mathbf{C}$ is called a multiplicative character if

$$
\phi(\sigma \tau)=\phi(\sigma) \phi(\tau) \text { for all } \sigma, \tau \in G .
$$

In the cyclic case multiplicative characters are eigenfunctions of the convolution operator and we have $n$ multiplicative characters, the Fourier complex exponentials, for example, see [1] for more details. The main problem with the non-abelian group, as opposed to the abelian one, is the lack of multiplicative characters. Multiplicative characters for any group $G$ are constant on its conjugacy classes.

Definition. A finite dimensional representation of a finite group $G$ is a group homomorphism

$$
\rho: G \mapsto G L(j, \mathbf{C})
$$

where $G L(j, \mathbf{C})$ denotes the general linear group of degree $j$, the set of all $j \times j$ invertible matrices. We refer to $j$ as the degree of the group representation. The field of complex numbers is denoted by $\mathbf{C}$.

Definition. Two group representations

$$
\rho_{1}: G \mapsto G L(j, \mathbf{C}) \text { and } \rho_{2}: G \mapsto G L(j, \mathbf{C})
$$

are said to be equivalent if there exists an invertible matrix $T \in M_{j \times j}(F)$ such that

$$
T \circ \rho_{1}(g) \circ T^{-1}=\rho_{2}(g)
$$

for all $g \in G$.

Every finite dimensional group representation is equivalent to a representation by unitary matrices. For more information on group representations see [2] for example.

Definition. Let $\hat{G}$ be the set of all (equivalence classes) of irreducible representations of the group $G$. Let $\rho \in \hat{G}$ be of degree $j$ and let $\phi \in \mathbf{C}^{n}$. Then the Fourier transform of $\phi$ at $\rho$ is the $j \times j$ matrix

$$
\hat{\phi}(\rho)=\sum_{s \in G} \phi(s) \rho(s) .
$$

The Fourier inversion formula, $s \in G$, is given by

$$
\phi(s)=\frac{1}{|G|} \sum_{\rho_{j} \in \hat{G}} d_{j} \operatorname{tr}\left(\rho_{j}\left(s^{-1}\right) \hat{\phi}\left(\rho_{j}\right)\right) .
$$

We alert the reader to an involution switch $s \rightarrow s^{-1}$ in the summand functions. We refer the reader to [3] for more details. Let $\mathbf{C}[G]$ be the algebra of complex valued functions on $G$ with respect to $G$-convolution. Let $\psi=\left(c_{0}, c_{1}, \cdots, c_{n-1}\right) \in \mathbf{C}^{n}$ and identify the function $\psi$ with its symbol

$$
\Psi=c_{0} \mathbf{1}+c_{1} g_{1}+\cdots+c_{n-1} g_{n-1} \in \mathbf{C}[G] .
$$

Let $\psi$ and $\phi$ be two elements in $\mathbf{C}^{n}$. We have a natural identification

$$
\psi * \phi \mapsto \Psi \Phi
$$

understood with respect to the induced group algebra multiplication. We have a non-abelian version of the classical $z$ transform. The action of $\psi$ on $\phi$ through $G$-convolution is captured by the matrix multiplication by the $G$-circulant matrix $C_{G}(\psi)$, in particular

$$
\psi * \phi=C_{G}(\psi) \phi
$$

The character of a group representation $\rho$ is the complex valued function

$$
\chi: G \rightarrow \mathbf{C}
$$

defined by

$$
\chi(g)=\operatorname{tr}(\rho(g)) .
$$

For all $g \in G$ the quantity $\chi(g)$ is a sum of complex roots of unity. Moreover, we have $\chi\left(g^{-1}\right)=\overline{\chi(g)}$ 
for all $g \in G$. A character is called irreducible if the underlying group representation is irreducible. We define an inner product on the space of class functions, functions on $G$ that are constant its conjugacy classes

$$
\langle\chi, \theta\rangle=\frac{1}{|G|} \sum_{g \in G} \chi(g) \overline{\theta(g)} .
$$

Note that a character is a class function. We have as many irreducible characters as there are conjugacy classes of $G$. If $G$ is abelian, then we have $n$ irreducible characters. With respect to the usual inner product we have

$$
\left\langle\chi_{i}, \chi_{j}\right\rangle=\delta_{i j}
$$

where $\delta_{i, j}$ is the Kronecker delta. Irreducible characters form a basis for the space of class functions on $G$.

Definition. Let $\Psi=c_{0} \mathbf{1}+c_{1} g_{1}+\cdots+c_{n-1} g_{n-1} \in \mathbf{C}[G]$. The adjoint of $\Psi$, denoted by $\Psi^{*}$, in the group algebra $\mathbf{C}[G]$ is the element

$$
\Psi^{*}=\bar{c}_{0} \mathbf{1}+\bar{c}_{1} g_{1}^{-1}+\cdots+\bar{c}_{n-1} g_{n-1}^{-1} .
$$

Associate $\Psi$ and $\Psi^{*}$ with the corresponding functions $\psi$ and $\psi^{*}$. We collect a few simple facts. Let $C_{G}^{*}(\psi)$ be the adjoint of the $G$-circulant matrix $C_{G}(\psi)$. Then we have $C_{G}^{*}(\psi)=C_{G}\left(\psi^{*}\right)$. The matrix $C_{G}(\psi)$ is normal if and only if $\Psi^{*} \Psi=\Psi \Psi^{*}$ and selfadjoint if and only if $\Psi=\Psi^{*}$.

The Fourier transform gives us a natural isomorphism

$$
\mathbf{C}[G] \Rightarrow M(\hat{G})
$$

where

$$
M(\hat{G})=M_{d_{1} \times d_{1}}(\mathbf{C}) \oplus M_{d_{2} \times d_{2}}(\mathbf{C}) \oplus \cdots \oplus M_{d_{r} \times d_{r}}(\mathbf{C})
$$

with $d_{1}^{2}+d_{2}^{2}+\cdots+d_{r}^{2}=n$. A typical element of $\mathbf{C}^{n}$ is a complex valued function

$$
\psi=\left(c_{0}, c_{1}, \cdots, c_{n-1}\right)
$$

and the typical element of $M(\hat{G})$ is the direct sum of Fourier transforms

$$
\hat{\phi}\left(\rho_{1}\right) \oplus \hat{\phi}\left(\rho_{2}\right) \oplus \cdots \oplus \hat{\phi}\left(\rho_{r}\right) .
$$

Fourier transform turns convolution into (matrix) multiplication

$$
\widehat{\psi * \phi}=\bigoplus_{j=1}^{r} \hat{\psi}_{j} \hat{\phi}_{j}=\hat{\psi} \hat{\phi}
$$

In the abelian setting the Fourier transform is a unitary linear transformation (proper scaling required). In the non-abelian setting we recapture this property if we define the right inner product on the space $M(\hat{G})$. We will provide more details on this later on. Let $\phi \in \mathbf{C}^{n}$ and define for $s \in G$

$$
\phi_{j}(s)=\frac{d_{j}}{|G|} \operatorname{tr}\left(\rho_{j}\left(s^{-1}\right) \hat{\phi}\left(\rho_{j}\right)\right) .
$$

Note $\phi=\sum_{j=1}^{r} \phi_{j}$. We are able to decompose a function $\phi$ into a sum of $r$ functions which is the number of conjugacy classes of $G$.

Every group $G$ admits a trivial irreducible representation $\rho_{1}$ for which $\rho_{1}(s)=1$ for all $s \in G$. For $\phi \in \mathbf{C}^{n}$ the Fourier transform of $\phi$ at $\rho_{1}$ is given by

and

$$
\hat{\phi}\left(\rho_{1}\right)=\sum_{s \in G} \phi(s)
$$

$$
\phi_{1}(s)=\frac{1}{|G|} \sum_{s \in G} \phi(s)
$$

Thus the constant mean function is always represented in our decomposition. The decomposition $\phi=\sum_{j=1}^{r} \phi_{j}$ 
must be orthogonal. The following can be, for example, found in [4]. The notation $\|\cdot\|_{F}$ refers to the Frobenius norm.

Proposition 1.1. Let $\phi_{j}$ be given as above. We have

$$
\left\langle\phi_{i}, \phi_{j}\right\rangle=\frac{d_{j}}{|G|}\left\|\hat{\phi}\left(\rho_{j}\right)\right\|_{F}^{2} \delta_{i, j} .
$$

Corollary 1.1. Let $\phi \in \mathbf{C}^{n}$ then

$$
\|\phi\|^{2}=\frac{1}{|G|} \sum_{j=1}^{r} d_{j}\left\|\hat{\phi}\left(\rho_{j}\right)\right\|_{F}^{2}
$$

Equip the space $M(\hat{G})$ with the following inner product. Let $\mathbf{v}=\hat{\phi}\left(\rho_{1}\right) \oplus \hat{\phi}\left(\rho_{2}\right) \oplus \cdots \oplus \hat{\phi}\left(\rho_{r}\right)$ and $\mathbf{w}=\hat{\zeta}\left(\rho_{1}\right) \oplus \hat{\zeta}\left(\rho_{2}\right) \oplus \cdots \oplus \hat{\zeta}\left(\rho_{r}\right)$. Then

$$
\left\langle\mathbf{v} \bullet_{F} \mathbf{w}\right\rangle=\frac{d_{1}}{|G|} \operatorname{tr}\left(\hat{\phi}\left(\rho_{1}\right) \hat{\zeta}^{*}\left(\rho_{1}\right)\right)+\frac{d_{2}}{|G|} \operatorname{tr}\left(\hat{\phi}\left(\rho_{2}\right) \hat{\zeta}^{*}\left(\rho_{2}\right)\right)+\cdots+\frac{d_{r}}{|G|} \operatorname{tr}\left(\hat{\phi}\left(\rho_{r}\right) \hat{\zeta}^{*}\left(\rho_{r}\right)\right)
$$

whete $\hat{\zeta}^{*}(\rho)$ denotes the adjoint of $\hat{\zeta}(\rho)$.

Corollary 1.2. The Fourier transform is a unitary transformation from $\mathbf{C}^{n}$ onto $\left(M(\hat{G}), \bullet_{F}\right)$.

For more information of non-abelian Fourier transform see the works of [5]-[11], for example.

\section{Main Results}

Consider for a moment $l^{2}(G)$, where $G$ is a cyclic group of size $n$. Given a vector $\phi \in l^{2}(G)$, we can writ

$$
\phi=\sum_{j=1}^{n} c_{j} \phi_{j}
$$

where the Fourier complex exponentials $\left\{\phi_{j}\right\}$ are orthonormal vectors and $\left\{c_{j}\right\}_{j=1}^{n}$ are the Fourier coefficients. Being multiplicative characters for $G$, these functions $\left\{\phi_{j}\right\}_{j=1}^{n}$ are $G$-decorrelated, in particular, for $i \neq j$,

$$
\sum_{t \in G} \bar{\phi}_{i}(t) \phi_{j}(t+\tau)=0 \text { for all } \tau \in G .
$$

This important property makes the Fourier exponentials vital in signal analysis. The need for time shift de-correlation or spatial shift de-correlation is reflected in the cyclic group structure of $G$.

We extend these observations to non-abelian groups $G$, recall that finite abelian groups are direct sums of cyclic groups. We say that two vectors $\psi, \phi$ in $l^{2}(G)$ are $G$-decorrelated if

$$
\sum_{t \in G} \bar{\psi}(t) \phi(t \tau)=0 \text { for all } \tau \in G .
$$

We observe that even in the non-abelian case the linearly independent multiplicative characters are $G$-decorrelated as the following simple observation reveals

$$
\sum_{j=1}^{r} \bar{\psi}(t) \phi(t \tau)=\phi(\tau) \sum_{j=1}^{r} \bar{\psi}(t) \phi(t)=0
$$

as linearly independent multiplicative characters are orthogonal.

Definition. For given vectors $\psi, \phi \in l^{2}(G)$ the $G$ cross-correlation function is defined by

$$
R_{\psi, \phi}(\tau)=\sum_{t \in G} \bar{\psi}(t) \phi(t \tau) .
$$

Note that $\psi$ and $\phi$ are $G$-decorrelated if and only if $R_{\psi, \phi} \equiv 0$. Recall we view the following three objects $\mathbf{C}[G], l^{2}(G)$ and $\mathbf{C}^{n}$ as isomorphic vector spaces.

Lemma 2.1. Consider $\psi, \phi \in l^{2}(G)$ and the corresponding $\Psi, \Phi \in \mathbf{C}[G]$. Then we have

$$
R_{\psi, \phi}=\Psi^{*} \Phi=C_{G}^{*}(\psi) \phi .
$$


Proof: We have

$$
R_{\psi, \phi}(\tau)=\sum_{t \in G} \bar{\psi}(t) \phi(t \tau)=\sum_{s \in G} \bar{\psi}\left(s \tau^{-1}\right) \phi(s)=\sum_{s \in G} \overline{\tilde{\psi}}\left(\left(s \tau^{-1}\right)^{-1}\right) \phi(s)=\sum_{s \in G} \overline{\tilde{\psi}}\left(\left(\tau s^{-1}\right)\right) \phi(s)=\Psi^{*} \Phi
$$

where $\tilde{\psi}(s)=\psi\left(s^{-1}\right)$.

Corollary 2.1. Let $\hat{\psi}, \hat{\phi}$ be the Fourier transforms of $\psi$ and $\phi$ respectively. Then we have

$$
\hat{R}_{\psi, \phi}=\hat{\psi}^{*} \hat{\phi} .
$$

Thus functions $\psi, \phi \in l^{2}(G)$ are $G$-decorrelated if and only if

$$
\hat{\psi}^{*} \hat{\phi}=0 \text {. }
$$

Corollary 2.2. Let $\psi, \phi \in l^{2}(G)$. Then $\psi$ and $\phi$ are $G$-decorrelated if and only if

$$
\phi \in \operatorname{Ker}\left(C_{G}^{*}(\psi)\right) \text {. }
$$

Observe that if $\phi$ is a multiplicative character then

$$
R_{\phi, \phi}=\langle\phi, \phi\rangle \phi .
$$

However, these are not the only functions with this property, i.e. $R_{\phi, \phi}$ is a multiple of $\phi$. In fact, we have the following, note that $\lambda$ below could be complex.

Lemma 2.2. Let $\phi \in l^{2}(G)$. Then

$$
R_{\phi, \phi}=\lambda \phi
$$

if and only if

$$
\hat{\phi}=\hat{\phi}\left(\rho_{1}\right) \oplus \hat{\phi}\left(\rho_{2}\right) \oplus \cdots \oplus \hat{\phi}\left(\rho_{\mathrm{r}}\right)
$$

with $\hat{\phi}\left(\rho_{j}\right)=\lambda Q_{j}$, where $Q_{j}$ is a projection matrix for all $j \in\{1,2, \cdots, r\}$. Note $\lambda$ in independent of $j$, but $Q_{j}$ can depend on $j$.

Proof: Using Corollary 2.1, the function $\phi$ has the property $R_{\phi, \phi}=\lambda \phi$ if and only if

$$
\hat{\phi}^{*}\left(\rho_{j}\right) \hat{\phi}\left(\rho_{j}\right)=\lambda \hat{\phi}\left(\rho_{j}\right)
$$

for all $j \in\{1,2, \cdots, r\}$. Observe that, as a result, the matrix $\hat{\phi}\left(\rho_{j}\right)$ has to be normal. Therefore, we can orthogonally diagonalize $\hat{\phi}\left(\rho_{j}\right)$ with the diagonal matrix $D_{j}=\left[d_{j}(i, i)\right]_{i=1}^{d_{j}}$. Now the above matrix equality translates to the following

$$
d_{j}(i, i) \overline{d_{j}(i, i)}=\lambda d_{j}(i, i) \text { for all } i \in\left\{1,2, \cdots, d_{j}\right\}
$$

which forces all the non-zero diagonal entries of $Q_{j}$ to be the same. This is exactly the claim that $\hat{\phi}\left(\rho_{j}\right)$ is a multiple of some projection matrix $Q_{j}$ that could depend of $j$.

We say a set of functions $\left\{\alpha_{j}\right\}_{j=1}^{m}$ in $l^{2}(G)$ is $G$-orthogonal if

$$
R_{\alpha_{i}, \alpha_{j}}=\lambda_{i} \alpha_{i} \delta_{i, j}
$$

where $\delta_{i, j}$ is the Kronecker delta and $\lambda_{i} \in \mathbf{C}$.

Theorem 2.1. Let $\phi \in l^{2}(G)$ and $\left\{\chi_{j}\right\}_{j=1}^{r}$ be the set of all irreducible characters of $G$. Then we have

$$
\phi=\sum_{j=1}^{r} \phi_{j}
$$

where

$$
\phi_{j}=\frac{d_{j}}{|G|} \tilde{\chi}_{j} * \phi=\frac{d_{j}}{|G|} C_{G}\left(\tilde{\chi}_{j}\right) \phi
$$

and the set of functions $\left\{\phi_{j}\right\}_{j=1}^{r}$ is $G$-decorrelated. If $\chi_{j}$ is a multiplicative character, $d_{j}=1$, then 


$$
\phi_{j}=\left\langle\phi, \tilde{\chi}_{j}\right\rangle \tilde{\chi}_{j} .
$$

Note that if $\phi$ and $\chi_{j}$ are real valued then so is the corresponding $\phi_{j}$. Moreover, the set $\left\{\chi_{j}\right\}_{j=1}^{r}$ is $G$-orthogonal.

Proof: Recall

$$
\phi=\sum_{j=1}^{r} \phi_{j} \text { where } \phi_{j}(s)=\frac{d_{j}}{|G|} \operatorname{tr}\left(\rho_{j}\left(s^{-1}\right) \phi\left(\rho_{j}\right)\right) .
$$

Now define an (orthogonal) projection $P_{j}$ on $\mathbf{C}^{n}$ by the following action, $\phi \in \mathbf{C}^{n}$

$$
P_{j}(\phi)=\phi_{j} \text {. }
$$

The action of the linear operator $P_{j}$ in the Fourier domain is given by the (matrix) multiplication by the vector

$$
0 \oplus \cdots \oplus 0 \oplus \mathbf{I}_{j} \oplus 0 \oplus \cdots \oplus 0
$$

where the $d_{j} \times d_{j}$ idenity matrix $\mathbf{I}_{j}$ is in the $j$ th position. The inverse Fourier transform of this vector is the function (evaluated at $g \in G$ )

$$
\frac{d_{j}}{|G|} \operatorname{tr}\left(\rho_{j}\left(g^{-1}\right)\right)=\frac{d_{j}}{|G|} \chi_{j}\left(g^{-1}\right)=\frac{d_{j}}{|G|} \overline{\chi_{j}(g)} .
$$

Therefore for all $\phi \in \mathbf{C}^{n}$ we have

$$
P_{j}(\phi)=\frac{d_{j}}{|G|} \tilde{\chi}_{j} * \phi
$$

where $\tilde{\chi}_{j}(g)=\operatorname{tr}\left(\rho_{j}\left(g^{-1}\right)\right)$ is the (inverted) character of the irreducible representation $\rho_{j}$. Now Proposition 1.1, Lemma 2.1 and Corollary 2.1 can be used to show that $\phi_{i}$ and $\phi_{j}$ are $G$-decorrelated. Using Lemma 2.2 we conclude that the set $\left\{\chi_{j}\right\}_{j=1}^{r}$ is $G$-orthogonal.

It is important to note that if ${ }^{j=1} \chi_{j}$ is not a multiplicative character, then

$$
\phi_{j} \neq\left\langle\phi, \tilde{\chi}_{j}\right\rangle \tilde{\chi}_{j}
$$

in general.

In order to obtain the above $G$-decorrelated decomposition one does not need to know explicitly the irreducible group representations, just the irreducible characters for the group $G$. For any group $G$ these (irreducible) characters are much easier to find than the corresponding irreducible group representations. This alone makes the above decomposition amicable for applications. Also note that in a case of multiplicative character $\chi_{j}$, the corresponding decomposition function $\phi_{j}$ is a multiple of the (inverted) character $\tilde{\chi}_{j}$.

However, in the case of (irreducible) character $\chi_{j}$ stemming from a higher dimensional irreducible representation, this is no longer the case. The intuitive interpretation of the function $\phi_{j}$ then becomes more difficult.

Corollary 2.3. Let $U=\left\{j_{1}, j_{2}, \cdots, j_{u}\right\}$ be a subset of $\{1,2, \cdots, r\}$. Then

$$
\sum_{j \in U} \phi_{j}=C_{G}\left(\tilde{\chi}_{U}\right) \phi
$$

where

$$
\tilde{\chi}_{U}=\sum_{j \in U} \frac{d_{j}}{|G|} \tilde{\chi}_{j}
$$

In the cyclic case we can talk about frequencies in the context of the Fourier complex exponentials. As a result, we can design filters, that can isolate specific frequencies and block others. In the non-abelian case this becomes less clear as the concept of frequencies is lost in the irreducible characters.

We can go further and obtain a $G$-decorrelated decomposition of any function $\phi \in l^{2}(G)$ that consists of $n$ summands. Moreover, this $G$-decorrelated decomposition is obtained by orthogonal projections. However, 
the drawback is that we have to know the irreducible representations of the group $G$ and not just the irreducible characters.

Theorem 2.2. Let $\phi \in l^{2}(G)$ and let $\left\{\rho_{j}\right\}$ be the set of all irreducible representations of $G, \rho_{j}$ has size $d_{j}$. Let $\rho_{j}(k, l)$ be the $(k, l)$ entry in the $d_{j} \times d_{j}$ matrix of $\rho_{j}$. Consider the (involuted) function $\rho_{j}(k, l)\left(g^{-1}\right) \in l^{2}(G)$. Then $\phi$ can be written as a $G$-decorrelated sum of vectors $\left\{\phi_{j}(k, l)\right\}_{j=1}^{n}$ where

$$
\phi=\sum_{j=1 k, l=1}^{n} \sum_{j}^{d_{j}} \phi_{j}(k, l)
$$

where

$$
\phi_{j}(k, l)=\frac{d_{j}}{|G|}\left\langle\phi, \rho_{j}(k, l)\right\rangle \rho_{j}(k, l) .
$$

Moreover, the (diagonal) set of functions $\left\{\rho_{j}(k, k)\right\}_{j=1, \cdots, r ; k=1, \cdots, d_{j}}$ is $G$-orthogonal.

Proof: We invoke the Schur's orthogonality relations, see [12], for example. With notation as in Theorem 2.1, we observe, using the Schur's orthogonality relations

$$
\operatorname{Im}\left(P_{j}\right)=\operatorname{span}\left\{\rho_{j}(k, l) \mid k, l \in\left\{1, \cdots, d_{j}\right\}\right\}
$$

and conclude, using Proposition 1.1, the functions

$$
\left\{\sqrt{\frac{d_{j}}{|G|}} \rho_{j}(k, l) \mid k, l \in 1, \cdots, d_{j}\right\}
$$

form an orthonormal basis for $\operatorname{Im}\left(P_{j}\right)$. Therefore, we have

$$
\tilde{\chi}_{j} * \phi=\sum_{k, l}\left\langle\phi, \rho_{j}(k, l)\right\rangle \rho_{j}(k, l) .
$$

Note that

$$
\hat{\rho}_{j}(k, l)=0 \oplus \cdots \oplus 0 \oplus E_{j}(l, k) \oplus 0 \oplus \cdots \oplus 0
$$

where $E_{j}(l, k)$ is a matrix whose entries are all zero except $E_{j}(l, k)=1$. Therefore

$$
\hat{\rho}_{i}(k, l) \hat{\rho}_{j}(s, t)=0
$$

unless $i=j$ and $(k, l)=(s, t)$. Now using Lemma 2.2 we conclude that the (diagonal) set of functions $\left\{\rho_{j}(k, k)\right\}_{j=1, \cdots, r ; k=1, \cdots, d_{j}}$ is $G$-orthogonal.

Note the (non-diagonal) set of functions.

$\left\{\rho_{j}(k, l)\right\}_{j=1, \cdots, r ; k, l=1, \cdots, d_{j}}$ is not necessarily $G$-orthogonal. Also, unlike the irreducible characters, we have $\rho_{j}(k, l)\left(g^{-1}\right) \neq \overline{\rho_{j}(k, l)}(g)$ in general.

\section{Example: The Symmetric Group $S_{3}$}

We will consider the symmetric group $S_{3}$ in our example. The group $G=S_{3}$ consists of elements

$$
\begin{gathered}
g_{0}=(1) ; g_{1}=(12) ; g_{2}=(13) \\
g_{3}=(23) ; g_{4}=(123) ; g_{5}=(132) .
\end{gathered}
$$

The group $S_{3}$ has three conjugacy classes

$$
\left\{g_{0}\right\},\left\{g_{1}, g_{2}, g_{3}\right\},\left\{g_{4}, g_{5}\right\} .
$$

We have three irreducible representations, two of which are one dimensional, $\rho_{1}$ is the identity map, $\rho_{2}$ is 
the map that assigns the value of 1 if the permutation is even and the value of -1 if the permutation is odd. Finally, we have $\rho_{3}$, the two dimensional irreducible representation of $S_{3}$, defined by the following assignment

$$
\begin{gathered}
g_{0} \mapsto\left(\begin{array}{cc}
1 & 0 \\
0 & 1
\end{array}\right) ; g_{1} \mapsto\left(\begin{array}{cc}
0 & 1 \\
1 & 0
\end{array}\right) \\
g_{2} \mapsto\left(\begin{array}{cc}
0 & \mathrm{e}^{2 \pi i / 3} \\
\mathrm{e}^{-2 \pi / 3} & 0
\end{array}\right) ; g_{3} \mapsto\left(\begin{array}{cc}
0 & \mathrm{e}^{-2 \pi i / 3} \\
\mathrm{e}^{2 \pi i / 3} & 0
\end{array}\right) \\
g_{4} \mapsto\left(\begin{array}{cc}
\mathrm{e}^{2 \pi i / 3} & 0 \\
0 & \mathrm{e}^{-2 \pi i / 3}
\end{array}\right) ; g_{5} \mapsto\left(\begin{array}{cc}
\mathrm{e}^{-2 \pi i / 3} & 0 \\
0 & \mathrm{e}^{2 \pi i / 3}
\end{array}\right) .
\end{gathered}
$$

The irreducible characters of $S_{3}$ are given by

$$
\begin{gathered}
\chi_{1}=(1,1,1,1,1,1)^{\mathrm{T}} \\
\chi_{2}=(1,-1,-1,-1,1,1)^{\mathrm{T}} \\
\phi_{3}=(2,0,0,0,-1,-1)^{\mathrm{T}}
\end{gathered}
$$

where $\chi_{1}$ and $\chi_{2}$ are also multiplicative characters. Moreover, we have

$$
\begin{aligned}
& \rho_{3}(1,1)=\left(1,0,0,0, \mathrm{e}^{-2 \pi i / 3}, \mathrm{e}^{2 \pi i / 3}\right)^{\mathrm{T}} \\
& \rho_{3}(1,2)=\left(0,1, \mathrm{e}^{-2 \pi i / 3}, \mathrm{e}^{2 \pi \mathrm{i} / 3}, 0,0\right)^{\mathrm{T}} \\
& \rho_{3}(2,1)=\left(0,1, \mathrm{e}^{2 \pi i / 3}, \mathrm{e}^{-2 \pi \mathrm{i} / 3}, 0,0\right)^{\mathrm{T}} \\
& \rho_{3}(2,2)=\left(1,0,0,0, \mathrm{e}^{2 \pi \mathrm{i} / 3}, \mathrm{e}^{-2 \pi i / 3}\right)^{\mathrm{T}} .
\end{aligned}
$$

Observe that all three irreducible characters are real valued and hence all the decomposition functions $\left\{\phi_{j}\right\}_{j=1}^{r}$ are also real valued if $\phi$ is real valued as well. The $G$-convolution by a function $\psi \in l^{2}(G)$ can be induced by a $G$-circulant matrix $C_{G}(\psi)$ given by

$$
C_{G}(\psi)=\left(\begin{array}{llllll}
\mathbf{c}_{0} & \mathbf{c}_{1} & \mathbf{c}_{2} & \mathbf{c}_{3} & \mathbf{c}_{4} & \mathbf{c}_{5} \\
\mathbf{c}_{1} & \mathbf{c}_{0} & \mathbf{c}_{4} & \mathbf{c}_{5} & \mathbf{c}_{2} & \mathbf{c}_{3} \\
\mathbf{c}_{2} & \mathbf{c}_{5} & \mathbf{c}_{0} & \mathbf{c}_{4} & \mathbf{c}_{3} & \mathbf{c}_{1} \\
\mathbf{c}_{3} & \mathbf{c}_{4} & \mathbf{c}_{5} & \mathbf{c}_{0} & \mathbf{c}_{1} & \mathbf{c}_{2} \\
\mathbf{c}_{4} & \mathbf{c}_{3} & \mathbf{c}_{1} & \mathbf{c}_{2} & \mathbf{c}_{5} & \mathbf{c}_{0} \\
\mathbf{c}_{5} & \mathbf{c}_{2} & \mathbf{c}_{3} & \mathbf{c}_{1} & \mathbf{c}_{0} & \mathbf{c}_{4}
\end{array}\right) .
$$

and specifically, note that $\tilde{\chi}_{j}(g)=\bar{\chi}_{j}(g)=\chi_{j}(g)$,

$$
C_{G}\left(\chi_{1}\right)=\left(\begin{array}{llllll}
1 & 1 & 1 & 1 & 1 & 1 \\
1 & 1 & 1 & 1 & 1 & 1 \\
1 & 1 & 1 & 1 & 1 & 1 \\
1 & 1 & 1 & 1 & 1 & 1 \\
1 & 1 & 1 & 1 & 1 & 1 \\
1 & 1 & 1 & 1 & 1 & 1
\end{array}\right) .
$$




$$
\begin{aligned}
C_{G}\left(\chi_{2}\right) & =\left(\begin{array}{cccccc}
1 & -1 & -1 & -1 & 1 & 1 \\
-1 & 1 & 1 & 1 & -1 & -1 \\
-1 & 1 & 1 & 1 & -1 & -1 \\
-1 & 1 & 1 & 1 & -1 & -1 \\
1 & -1 & -1 & -1 & 1 & 1 \\
1 & -1 & -1 & -1 & 1 & 1
\end{array}\right) . \\
C_{G}\left(\chi_{3}\right) & =\left(\begin{array}{cccccc}
2 & 0 & 0 & 0 & -1 & -1 \\
0 & 2 & -1 & -1 & 0 & 0 \\
0 & -1 & 2 & -1 & 0 & 0 \\
0 & -1 & -1 & 2 & 0 & 0 \\
-1 & 0 & 0 & 0 & -1 & 2 \\
-1 & 0 & 0 & 0 & 2 & -1
\end{array}\right) .
\end{aligned}
$$

Set $\phi=[\phi(0), \phi(1), \phi(2), \phi(3), \phi(4), \phi(5)]^{\mathrm{T}}$ and we obtain

$$
\begin{gathered}
\phi_{1}=\frac{1}{6} C\left(\chi_{1}\right) \phi=\frac{1}{6}\left\langle\phi, \chi_{1}\right\rangle \chi_{1} . \\
\phi_{2}=\frac{1}{6} C\left(\chi_{2}\right) \phi=\frac{1}{6}\left\langle\phi, \chi_{2}\right\rangle \chi_{2} . \\
\phi_{3}=\frac{1}{3} C\left(\chi_{3}\right) \phi .
\end{gathered}
$$

\section{Applications to Crossover Designs in Clinical Trials}

The application of non-abelian Fourier analysis has been studied extensively; we refer the reader to the works of [13] for example. However, we believe that the property of $G$-decorrelation among functions in $l^{2}(G)$ has to be further investigated. We have to capture a natural scenario where the underlying group structure $G$ is relevant to the corresponding $G$-decorrelation. One of the places this does appears is the crossover designs in clinical trials, in particular the William's $6 \times 3$ design with 3 treatments.

During a crossover trial each patient receives more than one treatments in a pre-specified sequence. Therefore, as a result, each subject acts as his or her own control. Each treatment is administered for a pre-selected time period. A so called washout period is established between the last administration of one treatment and the first administration of the next treatment. In this manner the effect of the preceding treatment should wear off, at least in principle. Still there will be some carry-over effects in all the specified treatment sequences, clearly starting with the second treatment. For more information on crossover designs in clinical trials see [14] or [15] for example.

In our example, we record the sum of all carry-over effects of the treatments in any given treatment sequence. We will follow the William's $6 \times 3$ design with 3 treatments $A, B$ and $C$. In particular we have 6 treatment sequences $A B C, A C B, B A C, B C A, C A B, C B A$. For example, suppose the order of treatment administration is $B C A$, with $B$ first. We decide to collect the sum of all carry-over effects of the treatments in this sequence (starting with the second one), $\lambda_{B C A}$. We observe the sequence $B C A$ as a permutation of the sequence $A B C$ by the permutation $g_{4}=(123)$, an element of the group $S_{3}$. Thus we can write $\lambda_{B C A}=\lambda\left(g_{4}\right)$. Similarly, a permutation sequence $A C B$ would result in $\lambda_{A C B}=\lambda\left(g_{3}\right)$.

It is here where we can capture the essence of $G$-decorrelation. We can start with some initial treatment order say $A B C$ and then administer crossover designs involving all 6 treatment permutations, denoted by $\phi_{1}$. Similarly, we could have started with a different initial combination of treatments, say BAC and then administer all 6 treatment permutations, denoted by $\phi_{2}$. Now it is natural to request for the data sequences $\phi_{1}$ and $\phi_{2}$ to be $G$-decorrelated, meaning that our data sequences are decorrelated even when we allow the initial treatment permutation to vary. 
Let us be specific and give a hypothetical example. Suppose we obtain a carryover sequence $\lambda=(2,3,1,0,4,5)^{\mathrm{T}}$, with the order of the elements respecting the group structure. Assume that the $\lambda$ values refer to the sums of all carryover effects of the treatments in the given sequence. For example $\lambda\left(g_{2}\right)=1$ and $\lambda\left(g_{4}\right)=4$. We now wish $G$-decorrelate the vector $\lambda$ over the group $S_{3}$. We obtain

$$
\begin{gathered}
\phi_{1}=\frac{1}{6}(15,15,15,15,15,15)^{\mathrm{T}}=(2.5,2.5,2.5,2.5,2.5,2.5)^{\mathrm{T}} . \\
\phi_{2}=\frac{1}{6}(7,-7,-7,-7,7,7)^{\mathrm{T}} \approx(1.17,-1.17,-1.17,-1.17,1.17,1.17)^{\mathrm{T}} . \\
\phi_{3}=\frac{1}{3}(-5,5,-1,-4,1,4)^{\mathrm{T}} \approx(-1.67,1.67,-0.33,-1.33,0.33,1.33)^{\mathrm{T}} .
\end{gathered}
$$

Observe $\left\|\phi_{1}\right\|_{2}=6.12,\left\|\phi_{2}\right\|_{2}=2.87$ and $\left\|\phi_{3}\right\|_{2}=3.01$. Observe that the function $\phi_{1}$ is a multiple of the multiplicative character $\chi_{1}$, and similarly, the function $\phi_{2}$ is a multiple of the multiplicative character $\chi_{2}$. However, the function $\phi_{3}$ is not a multiple of the (irreducible) character $\chi_{3}$, recall, the (irreducible) character $\chi_{3}$ is not a multiplicative character.

In the $G$-decorrelated sum the function $\phi_{1}$ represents the carry-over effect from the administration of the three treatments $A, B$ and $C$, reflecting the permutation independence in all of the carry-over effects from all 6 permutation options. The function $\phi_{2}$ reflects the sign permutation dependence, meaning how sensitive the carry-over effects are to switche from permuting two treatments versus three treatments. The interpretation of the function $\phi_{3}$ is more involved, the best is to view $\phi_{3}$ as $\phi_{3}=\phi-\phi_{1}-\phi_{2}$.

Let us now decompose the function $\phi_{3}$ further into a $G$-decorrelated sum. Now we have to use the irreducible representations of $S_{3}$ themselves, in particular the two dimensional irreducible representation $\rho_{3}$. We obtain the following

$$
\begin{aligned}
\phi_{3}(1,1) & =\frac{1}{3}\left\langle\lambda, \rho_{3}(1,1)\right\rangle \rho_{3}(1,1)=(-0.83-0.29 i, 0,0,0,0.17+0.87 i, 0.67-0.58 i)^{\mathrm{T}} . \\
\phi_{3}(1,2) & =\frac{1}{3}\left\langle\lambda, \rho_{3}(1,2)\right\rangle \rho_{3}(1,2)=(0,0.83-0.29 i,-0.17+0.87 i,-0.67-0.58 i, 0,0)^{\mathrm{T}} . \\
\phi_{3}(2,1) & =\frac{1}{3}\left\langle\lambda, \rho_{3}(2,1)\right\rangle \rho_{3}(2,1)=(0,0.83+0.29 i,-0.17-0.87 i,-0.67+0.58 i, 0,0)^{\mathrm{T}} . \\
\phi_{3}(2,2) & =\frac{1}{3}\left\langle\lambda, \rho_{3}(2,2)\right\rangle \rho_{3}(2,2)=(-0.83+0.29 i, 0,0,0,0.17-0.87 i, 0.67+0.58 i)^{\mathrm{T}} .
\end{aligned}
$$

As complex decompositions have little interpretation in our context, we can write a decomposition of $\phi_{3}$ with two vectors, $\phi_{3}(1,1)+\phi_{3}(2,2)$ and $\phi_{3}(1,2)+\phi_{3}(2,1)$,

$$
\begin{gathered}
\phi_{3}(1,1)+\phi_{3}(2,2)=(-1.67,0,0,0,0.33,1.33)^{\mathrm{T}} \\
\phi_{3}(1,2)+\phi_{3}(2,1)=(0,1.67,-0.33,-1.33,0,0)^{\mathrm{T}} .
\end{gathered}
$$

\section{References}

[1] Davis, P.J. (1979) Circulant Matrices. John Wiley and Sons.

[2] Dummit, D.S. and Foote, R.N. (1999) Abstract Algebra. John Wiley and Sons.

[3] Morrison, K.E. (1998) A Generalization of Circulant Matrices for Non-Abelian Groups. Private Communication. http://www.math.uh.edu/molshan/ftp/pub/proceed_cd.pdf

[4] Zizler, P. (2013) On Spectral Properties of Group Circulant Matrices. Pan American Mathematical Journal, $23,1-23$.

[5] Foote, R., Mirchandani, G., Rockmore, D., Healy, D. and Olson, T. (2000) A Wreath Product Group Approach to Signal and Image Processing: Part I-Multiresolution Analysis. IEEE Transaction in Signal Processing, 48, 102-132. http://dx.doi.org/10.1109/78.815483

[6] Healy, D., Mirchandani, G., Olson, T. and Rockmore, D. (1996) Wreath Products for Image Processing. Proceedings of the ICASSP, 6, 3582-3586. 
[7] Malm, E.J. (2005) Decimation-in-frequency Fast Fourier Transforms for the Symmetric Group. Thesis, Department of Mathematics, Harvey Mudd College.

[8] Maslen, D. and Rockmore, D. (1997) Generalized FFTs-A Survey of Some Recent Results. In: Finkelstein, L. and Kantor, W., Eds., Proceedings of the DIMACS Workshop on Groups and Computation, 183-237.

[9] Rockmore, D.N. (2003) Recent Progress and Applications in Group FFTs. NATO Advanced Study Institute on Computational Noncommutative Algebra and Applications.

[10] Sagan, B.E. (1991) The Symmetric Group: Representations, Combinatorial Algorithms, and Symmetric Functions. Wadsworth \& Brooks/Cole, Pacific Grove.

[11] Terras, A. (1999) Fourier Analysis on Finite Groups and Applications. Cambridge.

[12] Stankovic, R.S., Moraga, C. and Astola, J.T. (2005) Fourier Analysis on Finite Groups with Applications in Signal Processing and System Design. IEEE Press, John Wiley and Sons.

[13] Karpovsky, M.G. (1977) Fast Fourier Transforms on finite Non-Abelian Groups. IEEE Transaction on Computers, C-26, 1028-1030. http://dx.doi.org/10.1109/TC.1977.1674739

[14] Senn, S. (2002) Cross-Over Trials in Clinical Research. 2nd Edition, Wiley. http://dx.doi.org/10.1002/0470854596

[15] Chow, S.-C. and Liu, J.-P. (2013) Design and Analysis of Clinical Trials: Concepts and Methodologies. Wiley. 